\title{
Pneumonia research to reduce childhood mortality in the developing world
}

\author{
J. Anthony G. Scott, ${ }^{1,2}$ W. Abdullah Brooks, ${ }^{3}$ J.S. Malik Peiris, ${ }^{4}$ \\ Douglas Holtzman, ${ }^{5}$ and E. Kim Mulholland 6
}

\begin{abstract}
${ }^{1}$ Wellcome Trust/KEMRI Research Programme, Kilifi, Kenya. ${ }^{2}$ Nuffield Department of Clinical Medicine, University of Oxford, Oxford, United Kingdom. ${ }^{3}$ Centre for Health and Population Research, International Centre for Diarrhoea Disease Research, Dhaka, Bangladesh. ${ }^{4}$ Department of Microbiology, University Pathology Building, Queen Mary Hospital, University of Hong Kong, Hong Kong Special Administrative Region, People's Republic of China. 5Infectious Diseases Development, Global Health Program, Bill and Melinda Gates Foundation, Seattle, Washington, USA.

${ }^{6}$ Infectious Disease Epidemiology Unit, London School of Hygiene and Tropical Medicine, London, United Kingdom.
\end{abstract}

\begin{abstract}
Pneumonia is an illness, usually caused by infection, in which the lungs become inflamed and congested, reducing oxygen exchange and leading to cough and breathlessness. It affects individuals of all ages but occurs most frequently in children and the elderly. Among children, pneumonia is the most common cause of death worldwide. Historically, in developed countries, deaths from pneumonia have been reduced by improvements in living conditions, air quality, and nutrition. In the developing world today, many deaths from pneumonia are also preventable by immunization or access to simple, effective treatments. However, as we highlight here, there are critical gaps in our understanding of the epidemiology, etiology, and pathophysiology of pneumonia that, if filled, could accelerate the control of pneumonia and reduce early childhood mortality.
\end{abstract}

\section{Introduction}

Every year 1.9 million children under 5 years of age die from pneumonia (1). Indeed, it is the leading cause of child death in the world. Pneumonia is an acute illness in which the alveolar air spaces of the lung become inflamed and filled with fluid and white blood cells, giving rise to the appearance of consolidation on the chest radiograph. It can be caused by bacterial, viral, or parasitic infection as well as by noninfectious agents. Most severe cases of pneumonia are caused by bacteria, of which the most important are Streptococcus pneumoniae (pneumococcus) and Haemophilus influenzae. In developing countries, where patients are often treated without seeing a doctor, the WHO defines clinical pneumonia simply as an acute episode of cough or difficulty breathing associated with an increased respiratory rate (2).

Pneumonia is a disease of all ages, and in adult medical wards across the developing world it is one of the most common admission diagnoses. In contrast to the industrialized world, pneumonia is found characteristically in younger adults, who have a substantial inpatient mortality of $5 \%-23 \%$ (3). The pathogens causing pneumonia in children and adults are similar, and most respiratory pathogens are transmitted effectively between generations within households. In the United States, preventing pneumonia in children by vaccinating against pneumococcal disease has resulted in less pneumonia in adults (4). However, little is known about adult pneumonia in developing countries, and research is rare outside the context of emerging infections (3). There are thus considerable opportunities for pneumonia

Nonstandard abbreviations used: Hib, Haemophilus influenzae type b; IMCI, Integrated Management of Childhood Illness; PCV, pneumococcal conjugate vaccine; RSV, respiratory syncytial virus.

Conflict of interest: E.K. Mulholland is a member of the Global Alliance for Vaccines and Immunization's Hib Initiative. W.A. Brooks has conducted research funded by the Bill and Melinda Gates Foundation, but was not the direct recipient of the grant. J.A.G. Scott and E.K. Mulholland are members of the PneumoCarr consortium, which received funding from the Bill and Melinda Gates Foundation through its initiative "Grand Challenges in Global Health." The remaining authors have declared that no conflict of interest exists.

Citation for this article: J. Clin. Invest. 118:1291-1300 (2008). doi:10.1172/JCI33947. research on adults. However, in this Review, we concentrate on childhood pneumonia and specifically on research to reduce the unacceptable magnitude of child deaths from this disease.

Historically, pneumonia was the main cause of child death in developed countries, and in the United States in 1900, it is estimated that pneumonia killed 47 of every 1,000 children before the age of 5 years (5). Improvements in nutrition and living standards in the United States in the first 40 years of the 20th century led to a substantial reduction in pneumonia mortality well before antibiotics became available as an effective treatment (Figure 1 and refs. 6-8). However, in the low-income countries of Asia and Africa, pneumonia is still the main cause of child death. In developing countries, over one-quarter of children have an episode of clinical pneumonia each year throughout the first 5 years of their lives (9). On average, $2 \%-3 \%$ of children each year have pneumonia severe enough to require hospitalization, and many of these disease episodes are potentially fatal (9). Thus, for every 1,000 children born, about 100-150 episodes of severe pneumonia arise during the first 5 years of life, most during the first 2 years. Approximately $21 \%$ of child deaths are due to pneumonia (1), and many developing countries have mortality rates of 60-100 per 1,000 children under 5 years of age (10); this suggests that of every 1,000 children born alive, $12-20$ die from pneumonia before their fifth birthdays.

Although we know the number of children who die of pneumonia in developing countries, few of the causal factors leading to death have been elucidated. Mortality is associated with poverty and with malnutrition; critically, we do not yet know the extent to which it is caused by lack of access to health care. If access to care is inversely related to mortality, the impact of new diagnostics, medical treatments, and vaccines will be substantially constrained. Therefore, the first priority for pneumonia research is a better understanding of the epidemiology of fatal pneumonia. Increased understanding of this, as well as more detailed information about the etiology and pathophysiology of the disease, should guide new approaches to tackle the immense global problem of child deaths from pneumonia. 


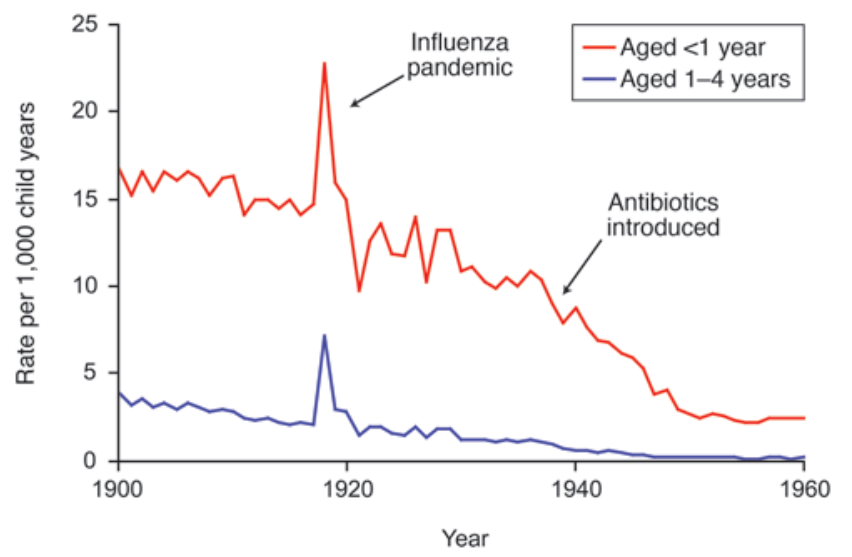

Figure 1

Mortality rate attributable to either pneumonia or influenza among children aged less than 5 years in the United States, 1900-1960. The combined annual rates of death per 1,000 child years from either pneumonia or influenza were compiled from U.S. government reports and stratified into two age groups $(6,7)$. Improvements in the rates of mortality from either pneumonia or influenza before the introduction of antibiotics were associated with major interventions to improve infant feeding and nutrition and with improved housing and reduced indoor air pollution.

\section{Diagnosis and etiology of pneumonia}

Conventional diagnosis of pneumonia consists of two stages: first, determining the syndrome by history, clinical examination, and chest radiology; and second, determining the etiology by microbiological, serological, and molecular tests. The WHO definition of clinical pneumonia described above is deliberately sensitive to maximize access to lifesaving antibiotics, but it has poor specificity. The WHO definition of severe pneumonia, which requires a hospital admission, is the presence of lower chest wall collapse (also known as lower chest wall indrawing; ref. 2). This also lacks specificity: lower chest wall collapse is found in cases of lower airway obstruction, bronchiolitis, and asthma as well as pneumonia. More recently, the WHO has also pioneered the standardized interpretation of chest X-rays to define bacterial pneumonia (11). This interpretation was designed as an epidemiologically specific endpoint to evaluate the efficacy of vaccines against bacterial pneumonia (12), but many children with clinical pneumonia and an abnormal radiograph do not satisfy the specific WHO criteria for radiological pneumonia (i.e., signs of alveolar consolidation and/or pleural effusion). Research to refine pneumonia classification, using clinical signs and a more sophisticated radiological interpretation, is a necessary prelude to future research on the etiology of the disease.

Empiric treatment guidelines. The primary rationale for etiological research in developing countries is to determine empiric treatment guidelines. Laboratories cannot determine etiology in sufficient time to influence management effectively, and in any case, few hospitals have the resources to investigate patients on an individual basis. Here, there are three challenges. First, it is difficult to obtain appropriate specimens from the lower respiratory tract. Children cannot normally produce sputum, and so the most useful etiologic data have been obtained in lung aspirate studies that provide microbiological evidence with the strongest claim on pneumonia causality (13). However, because lung aspirates are invasive, they are only conducted at a small number of research centers in developing countries $(14,15)$. Second, many pathogens that cause pneumonia are fastidious and require sophisticated laboratory culture systems for growth or replication. Third, existing tests for most pathogens that cause pneumonia are imperfect, and there is therefore no gold standard against which to test new diagnostics.

Isolation of bacteria from the blood of patients with clinical signs of lung infection is highly specific for bacterial pneumonia, but it has a sensitivity of less than $15 \%$ (12). Conventional diagnosis of viral pneumonia through cell culture or immunofluorescence techniques also has poor sensitivity (16). PCR can increase the sensitivity of pathogen detection (17-21), and multiplex PCR formats can improve throughput $(16,22)$. For example, the Respiratory MultiCode-PLx Assay (RMA; EraGen Biosciences) integrates multiplex PCR with microsphere flow cytometry to allow simultaneous identification of eight groups of respiratory virus (respiratory syncytial virus [RSV]; parainfluenza virus; influenza A and influenza $\mathrm{B}$; human rhinovirus; enteroviruses; metapneumovirus; adenovirus $\mathrm{B}$, adenovirus $\mathrm{C}$, and adenovirus $\mathrm{E}$; and coronaviruses). Compared with conventional diagnostic methods, this technique increases the number of pathogen-positive samples roughly 3 -fold (16).

Similarly, the mass tag technique links multiplex PCR for both bacterial and viral pathogens to the detection of stable organic molecules with unique molecular weights, known as mass tags (23). These molecules are coupled to the oligonucleotides used in PCR through UV-cleavable linkers and are detected with mass spectrometry following purification of amplification products. Because each oligonucleotide has a unique mass tag, and a positive signal is defined as the presence of paired mass tags, this gives increased confidence that the signal is the result of a specific amplification reaction. New microarray and mass spectrometry methods permit an even broader survey of potential pathogens (24-27). The challenge these technologies present to epidemiological research is to determine the causal attribution of organisms identified in blood or nasal secretions in the etiology of pneumonia.

Diagnostic tests to guide individual case management. For a new diagnostic test to be effective in the developing world it must be rapid, simple to execute, and inexpensive. For example, in adults, simple immunochromatographic strip tests can detect bacterial polysaccharides in urine and facilitate rapid diagnosis $(28,29)$. Unfortunately, this approach lacks specificity in children because, unlike adults, they often carry respiratory pathogens in their nasopharynx and therefore frequently excrete bacterial polysaccharides (30). For example, $60 \%-90 \%$ of children in developing countries are carriers of S. pneumoniae (31).

Numerous attempts have been made to identify biomarkers that can rapidly differentiate bacterial from viral pneumonia and thus focus antibiotic therapy on those most likely to respond. Serum levels of the acute phase proteins procalcitonin and C-reactive protein (CRP) are elevated in pneumonia, but their specificity, sensitivity, and predictive values vary widely in validation studies (32-34). Nonetheless, a CRP level of $40 \mathrm{mg} / \mathrm{dl}$ or greater, combined with a clinical diagnosis of pneumonia, identifies a greater proportion of pneumococcal pneumonias (defined as preventable by pneumococcal vaccination) than does clinical diagnosis alone (35). A number of cytokines and chemokines, including IL-1 receptor antagonist, IL-1 $\beta$, IL-6, IL-8, G-CSF, TNF- $\alpha$, and soluble triggering receptor expressed on myeloid cells (sTREM) have also shown diagnostic potential in severe bacterial infections, although none of these has yet been validated in the developing world for diagnosis of pneumonia (36-41).

Respiratory viruses and pneumonia with multiple etiologies. In industrialized countries, influenza strains other than H5N1 cause pneumonia 


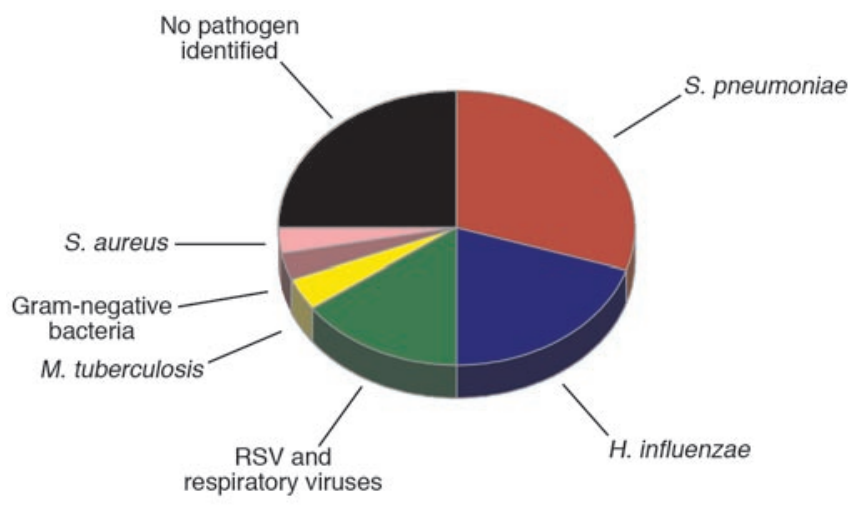

Figure 2

Etiology of severe pneumonia in children in developing countries. Qualitative representation of the combined results of vaccine probe analyses $(12,113)$, studies of blood and lung aspirate cultures (14, $111,153-155)$, and virus studies $(128,156)$ in predominantly HIVuninfected children. Note that children might be infected by two or more lung pathogens simultaneously. Single-etiology studies and studies of children in the developed world suggest the undiagnosed portion might be attributable, at least in part, to respiratory viruses (adenoviruses, influenza viruses, parainfluenza virus, human metapneumovirus, cytomegalovirus, rhinovirus, enteroviruses, and coronaviruses), Pneumocystis jirovecii, Mycoplasma pneumoniae, Chlamydia trachomatis, and Chlamydia pneumoniae. Etiology differs substantially in young infants (112), HIV-infected children (157), and malnourished children (15) as well as by region; for example, $S$. aureus accounted for onequarter of the cases of pneumonia in a large study in Chile (158).

and lower respiratory disease in children, often leading to hospitalization $(42,43)$. However, their role in mortality and morbidity in developing countries is poorly defined. In a study in rural Thailand, influenza virus was detected in approximately $10 \%$ of all patients hospitalized with pneumonia, with infants and those aged 75 years or older being at greatest risk (44). An underlying respiratory disease was found in $26 \%$ of older patients admitted with pneumonia caused by infection with the influenza virus, but underlying disease was uncommon in children under 5 years of age (44). Although bacteria and viruses can cause pneumonia independently, influenza and S. pneumoniae can interact to exacerbate severe pneumonia $(45,46)$, and at least one animal model provides evidence of lethal synergism (47). Specifically, the influenza neuraminidase protein facilitates pneumococcal invasiveness by stripping sialic acid from receptors on epithelial cells lining the respiratory tract. Pneumococci can attach to these receptors, enhancing their invasiveness. This viral contribution to the initiation of pathology often goes unrecognized. Conversely, preventing the pneumococcal complications of viral infections by pneumococcal vaccination can reduce the morbidity associated with pneumonia caused by infection with respiratory viruses (48).

The increased application of molecular methods of diagnosis has revealed that patients with acute respiratory infections (ARIs), including pneumonia, often have multiple viruses detected in the respiratory tract (49). The relative contributions of these pathogens to the genesis of respiratory pathology and their interactions with bacterial pathogens are clear opportunities for future research.

Pneumonia of unknown etiology and emerging lung infections. Studies of children in developing countries suggest that about half of all pneumonias are caused by either $H$. influenzae or S. pneumoniae (Figure 2). However, it is not always possible to define the caus- ative pathogen, and even with sophisticated laboratory techniques $25 \%-33 \%$ of cases of pneumonia worldwide are not attributed to any pathogen $(16,50)$. An unresolved question in the field of pneumonia research is the extent to which cases without a definable cause either reflect the poor sensitivity of current diagnostic tools for known pathogens or point to novel uncharacterized pneumonia pathogens. Indeed, the recent association of novel coronaviruses and rhinoviruses with respiratory disease in humans (51-53) highlights the need for an adaptable approach to the etiological investigation of pneumonia. It is likely that new pathogens will continue to emerge and that the etiological profile of pneumonia will alter considerably when the currently dominant pathogens, $H$. influenzae and S. pneumoniae, decline following vaccination.

The pathogens that best illustrate the challenge posed by newly emerging lung infections are the SARS coronavirus (SARS-CoV) and avian influenza H5N1. Both viruses cause pneumonia in children as well as adults. H5N1 influenza causes severe pneumonia in children, whereas the SARS-CoV causes milder disease in children, for reasons that remain obscure (54-56). The SARS epidemic illustrated the importance of surveillance for early recognition and rapid response to emerging pathogens. Indeed, the control of SARS was a triumph for global public health, and it was achieved by simple public health measures prior to the availability of vaccines and drugs. However, this success was possible only because the SARS-CoV is poorly transmissible in the first few days of the illness (57). Such favorable characteristics are unlikely to be replicated in a full-blown influenza pandemic.

In developed countries, vaccine control is likely to prove pivotal in defense against emerging pathogens that cause pneumonia (58). Indeed, intense effort is being directed to the development of vaccines against the animal coronaviruses that could be the precursors of a future SARS-like disease and also existing strains of avian influenza $\mathrm{H} 5 \mathrm{~N} 1$, such that in the event of human epidemic a vaccine might be more easily and more rapidly produced. However, selection of the antigens to which the vaccines should be targeted is proving difficult given the broad antigenic diversity of the animal coronaviruses (59) and the existing strains of avian influenza H5N1.

In developing countries, the inaccessibility of vaccines and antiviral drugs suggests that the only means of controlling outbreaks of emerging respiratory infections or an influenza pandemic will be simple public health measures such as social distancing, masks, and hand hygiene. Surprisingly, there are few studies on the efficacy of such interventions on the transmission of influenza or other respiratory viruses (60). Mathematical models are useful to predict the effectiveness of interventions at the population level, but these models are dependent on the accuracy of their transmission and intervention parameters. Such data can only come from well-designed, community-based epidemiological studies. With the tools of modern diagnostics, genomics, and immunology, it is now timely to revisit the classical Virus Watch studies of respiratory infections in families in the 1960s (61). Models and epidemiological studies should also consider heterogeneity in individual infectiousness, which has been observed in SARS, measles, and smallpox. Paradoxically, mathematical models predict that epidemics that arise out of human interactions of extraordinary infectiousness, i.e., superspreading events, might be especially susceptible to extinction and easier to curtail through individual control measures (62).

Both SARS and avian influenza H5N1 are zoonotic in origin, and this emphasizes the need for better understanding of the microbial ecology of domestic and wild animals and for closer collaboration 
among scientists in human, agricultural, and wildlife research. The elucidation of viral, genetic, and environmental determinants that permit or restrict the interspecies transmission of these pathogens is a major research challenge.

\section{Pathophysiology of and immunity to pneumonia}

The pathophysiology of pneumonia and immune regulation of the inflammatory response to lung infection are poorly understood, and few of the factors causing severe disease or death have been identified. Correction of hypoxia and zinc supplementation are two interventions that have proven efficacious, but research into the control of inflammation also offers considerable potential to improve outcomes.

Hypoxia. Severe childhood pneumonia is characterized by respiratory distress, which includes lower chest wall indrawing and frequently hypoxia. In young children, lower chest wall indrawing occurs because they have a soft sternum, horizontal ribs, and poor intercostal muscle development; when they try to generate greater negative pressure to inflate the lungs because of the presence of consolidation and lower airway obstruction, their lower chest wall collapses. Because airflow resistance increases inversely to the fourth power of the radius, small changes in airway diameter as a result of inflammation substantially reduce airflow. Hypoxia is thought to result primarily from ventilation/perfusion mismatch, although children with pneumonia in developing countries do respond to treatment with small amounts of oxygen (63). Without correction, hypoxia and acidosis set the stage for respiratory failure and death.

Estimates of the prevalence of hypoxia among children with pneumonia vary with hospital admission policies and with the effects of altitude. Up to $59 \%$ of admitted children are found to have a saturation of peripheral oxygen $\left(\mathrm{SPO}_{2}\right)$ lower than $90 \%$ (moderate hypoxia) and up to $29 \%$ have $\mathrm{SPO}_{2}$ lower than $70 \%$ (severe hypoxia) (64-66). Among Kenyan children hospitalized with pneumonia, those with hypoxia were four times more likely to die than those without hypoxia (64). The clinical signs of pneumonia identify hypoxia poorly in children $(64,65)$, and in Papua New Guinea, using pulse oximetry to guide oxygen therapy rather than using clinical signs alone reduced mortality by $35 \%$ (66). The WHO recommends providing oxygen, via either nasal prongs or catheter, to all children exhibiting any of the following signs: moderate hypoxia, cyanosis, inability to feed, or a respiratory rate greater than 60 breaths per minute (67). However, adoption of this approach in developing countries has been low. There are several reasons for this, including insufficient oxygen supply, misunderstanding of the specifications of pulse oximeters appropriate for developing countries, lack of cost-effectiveness data, and ignorance of the prevalence and distribution of hypoxemia $(67,68)$. Furthermore, most hospitals in developing countries purchase oxygen in cylinders, which is expensive, rather than investing in more economical oxygen concentrators $(67,69)$. The potential impact of this effective, low-cost intervention on child mortality deserves urgent investigation.

Vitamin A and zinc supplementation. Observational studies report that children with vitamin A deficiency have a 2-fold or higher risk of respiratory tract infection and death from pneumonia $(70,71)$. Although vitamin A supplementation reduces mortality among children under 2 years of age following infection with the measles virus (72), it has no apparent benefit in the treatment (73) or prevention $(74,75)$ of pneumonia. The observed association between pneumonia and vitamin A deficiency might therefore be attrib- utable to other confounding risk factors, such as zinc deficiency, which occurs in the same populations and is also associated with increased risk of acquiring respiratory infections (76). Indeed, zinc supplementation reduces the incidence of pneumonia by up to $40 \%$ and reduces the duration of hospitalization by $25 \%(77,78)$. Moreover, it can substantially reduce mortality in children under 2 years of age, primarily as a result of its effects on severe pneumonia (79); this reduction is sustained for children up to 5 years of age (80).

How zinc supplementation reduces the incidence and severity of pneumonia is not known, but there is evidence to suggest that it acts through the acute phase of the immune response to infection (81). The acute phase of an immune response to lung infection involves macrophages, lymphocytes, NK cells, and antibody-dependent cytotoxicity (82). Zinc decreases infection-related lung inflammation directly, and zinc deficiency enhances airway inflammation and cellular damage (83). In animal models, bacterial growth inhibition and host cell regeneration are both increased in the presence of zinc (84). Because these effects are pathogen independent, zinc supplementation offers substantial promise as a way to decrease child mortality due to pneumonia in developing countries, where limited resources constrain the determination of etiology.

A substantial constraint on the utility of zinc supplementation in preventing pneumonia is the relative paucity of data from Africa, where both malaria and HIV might modify its protective effect. A single study from Lesotho reported that zinc supplementation resulted in a modest reduction in pneumonia morbidity, but it was confined to children with severe protein-energy malnutrition (85). Elsewhere, a study from Zanzibar, which is holoendemic for malaria, reported a statistically nonsignificant $12 \%$ reduction in pneumonia-related mortality among children receiving zinc supplements (86), and a zinc supplementation trial in South Africa among children 6-60 months of age infected with HIV-1 reported no increase in HIV-1 viral load and a statistically significant $14 \%$ reduction in the incidence of diarrhea (87). Neither study, however, reported effects on pneumonia incidence.

The role of zinc in pneumonia treatment is also unclear, because results from hospital-based studies using different methodologies are inconsistent $(78,88-91)$. Furthermore, the use of zinc supplementation in outpatient settings, where most children with pneumonia are treated, has not been explored at all. In summary, zinc supplementation reduces pneumonia incidence prophylactically and reduces the risk of pneumonia-related mortality. More evidence is required to determine its acute effects as a treatment for pneumonia.

Innate immunity. In pneumonia research, acquired immunity has received considerable attention but the role of innate immunity, particularly relating to severe childhood pneumonia, has not. Animal models show that TLRs are essential for innate immunity to bacteria $(92,93)$ and viruses $(94,95)$, promoting cytokine-mediated inflammation. Cytokines and chemokines, including TNF- $\alpha$, IL-6, IFN- $\gamma$, IL-1 $\beta$, IL-4, IL-8, macrophage inflammatory protein-1 $\alpha$, and eotaxin, promote inflammation and pathogen clearance (96). Antiinflammatory cytokines such as IL-10 then reduce inflammation (Figure 3 and ref. 96). When inflammation is not balanced by antiinflammation, it causes lower airway obstruction and cellular damage $(96,97)$ and can even facilitate bacterial growth $(98)$. The severity of lung damage and high mortality caused by infection with H5N1 influenza and the 1918 pandemic strain of influenza might be explained by unbalanced inflammatory responses that can be induced by these viruses in vitro and in macaques $(99,100)$. 


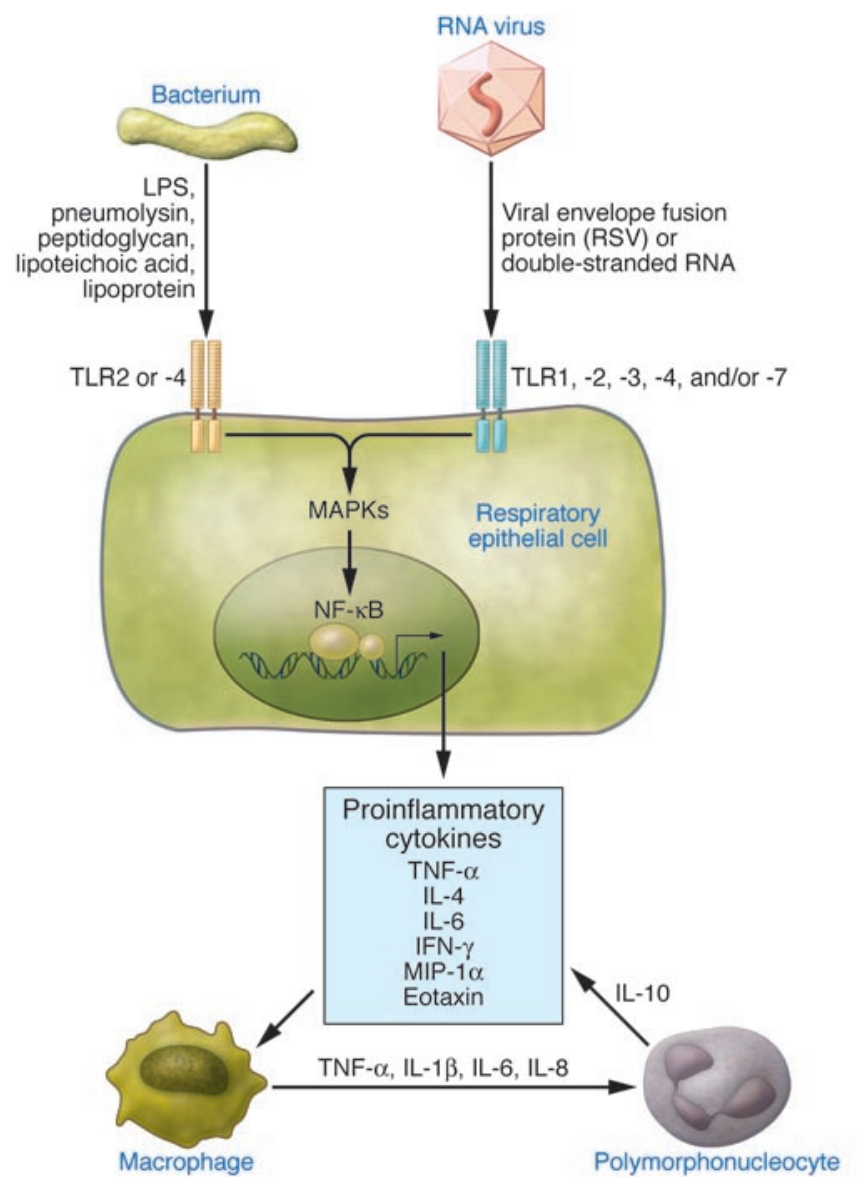

Figure 3

TLRs and the cytokine response to bacterial and viral pathogens. Gram-negative bacteria (e.g., E. coli), Gram-positive bacteria (e.g., $S$. pneumoniae), and RNA viruses attach to different receptors on the respiratory epithelium, triggering a proinflammatory cascade that results in activation of macrophages and polymorphonucleocytes. The latter, in turn, trigger antiinflammatory negative feedback to shut down inflammation. MIP-1 $\alpha$, macrophage inflammatory protein-1 $\alpha$.

Similar to zinc, TLRs and cytokines both modulate lung inflammation $(83,92,96)$. An understanding of the balance of their actions would help explain the mechanism of action of zinc and might lead to new interventions that could control inflammation, reduce severity, and prevent death.

\section{Preventing pneumonia through vaccination}

Immunization has enormous potential to reduce the burden of childhood deaths from pneumonia in developing countries. Vaccines are in development against several pathogens that cause pneumonia, including parainfluenza virus, Staphylococcus aureus, and Mycobacterium tuberculosis (101-103). Here we limit our discussion to vaccines against the most common pneumonia-causing pathogens: H. influenzae type b (Hib), pneumococcus, and RSV.

Conjugate vaccines against Hib and pneumococcus. Polysaccharideprotein conjugates illustrate how simple laboratory insights can be translated into substantial public health gain. The polysaccharides in the capsules of Hib and pneumococcus are the targets of mature humoral immunity and elicit T cell-independent responses (104).
Children can respond optimally to these antigens only after the first year of life, when their immune systems have matured (105, 106). However, in order to circumvent the limitations of the infant immune system, developers of polysaccharide-protein conjugates have used the 1929 observation of Avery and Goebel that conjugation of serum proteins to synthetic nonantigenic carbohydrates produced an antigenic compound that induced an immune response directed toward the carbohydrate component (107). The protein moiety stimulates $\mathrm{T}$ cell-dependent humoral immunity, which develops early in infancy, but the specificity of the response is focused on the polysaccharide moiety.

Polysaccharide-protein conjugate vaccines to prevent diseases caused by Hib or seven serotypes of pneumococcus were licensed in industrialized countries in 1990 and 2000, respectively, and have virtually eliminated disease caused by the targeted serotypes among immunized populations $(108,109)$. Early experience with the Hib vaccine in developing countries has confirmed its effectiveness (110). The use of these conjugate vaccines has also provided substantial insights into the epidemiology of pneumonia. For example, in The Gambia, results of early studies of blood and lung aspirate cultures suggested that Hib was the cause of approximately $7 \%$ of cases of pneumonia $(111,112)$. Later, in a randomized controlled trial, the observed efficacy of the Hib conjugate vaccine against radiologically confirmed pneumonia was $21 \%$, indicating that at least this fraction of pneumonia was caused by Hib (113). A subsequent trial in The Gambia of a 9-valent pneumococcal conjugate vaccine (PCV), so called because it contains capsular antigens of nine serotypes of pneumococcus, quantified the proportion of radiologically confirmed pneumonia caused by these pneumococcal serotypes at $37 \%$ (12); in The Gambia, Hib and nine serotypes of pneumococcus were the cause of over $50 \%$ of cases of radiologically confirmed pneumonia.

PCVs protect vaccinated children by inducing neutralizing IgG in serum (114). They also reduce the prevalence of nasopharyngeal carriage of the pneumococcal serotypes included in the vaccine (vaccine-type pneumococci; ref. 115), possibly because serum IgG seeps across the mucosal surface (116). The nasopharynx of young children is the principal natural reservoir of pneumococcus, and reduced carriage leads to decreased transmission and a decline in pneumococcal disease in unvaccinated individuals - herd protection. In America, two cases of vaccine-type pneumococcal disease have been prevented among nonvaccinated individuals for every one prevented directly by immunization (109).

The overall prevalence of nasopharyngeal carriage of pneumococcus after vaccination with PCV remains roughly constant as other serotypes take up the mucosal territory vacated by the pneumococcal serotypes included in the vaccine $(115,117)$. Most nonvaccine pneumococcal serotypes are less invasive than vaccine serotypes, but they can still cause disease, including pneumonia, and serotype-replacement disease has reduced the benefits of immunization by about one-sixth in the United States (109). Among Alaska Native children aged under 2 years, however, serotype-replacement disease has been more dramatic, reducing the direct benefits of vaccination by $50 \%$ (118). Increasing the vaccine valency would overcome this problem, although the complexity of conjugation and the 91 different potential serotypes limit the feasibility of this approach. Given evidence of the substantial direct protection afforded by PCVs, the Global Alliance on Vaccines and Immunization is supporting the introduction of the only commercially available formulation, 7-valent PCV, into several developing countries in 2008, 
with operational effectiveness studies due to be performed in The Gambia and Kenya. The relative magnitude of herd protection and serotype-replacement disease observed in these studies will be a strong determinant of the value of this program.

Other vaccines against pneumococcal pneumonia. Immunoepidemiological studies in developing countries suggest that childhood exposure through nasopharyngeal carriage and intermittent pneumococcal invasion generates immune responses to noncapsular antigens (119). This has led to the search for common surface proteins that could overcome the restricted serotype coverage of capsular polysaccharide PCVs. Candidate proteins include pneumolysin, two pneumococcal surface proteins that have a choline-binding function (PspA and PspC), a metal-binding transporter, pneumococcal surface adhesin A (PsaA), and two pneumococcal histidine triad proteins (PhtB and $\mathrm{PhtE}$ ). Each protein elicits antibodies that protect against systemic challenge in animal models, and protection is increased by combining proteins $(120,121)$. Reverse vaccinology - deriving antigens from the pneumococcal genome - has also revealed new surface-expressed antigens, including the newly identified pneumococcal pili (122). PspA elicits antibodies in humans that are protective in mice against fatal intravenous challenge with pneumococci, but there are as yet no reports of successful human protection using protein vaccines (123).

Intriguingly, mouse studies using inactivated, unencapsulated, whole-cell pneumococci as a vaccine have shown protection (124), which is abrogated in mice that lack $\mathrm{CD} 4^{+} \mathrm{T}$ cells congenitally and in mice depleted of $\mathrm{CD}^{+} \mathrm{T}$ cells using monoclonal antibodies, suggesting that protection against pneumococcal colonization is mediated by $\mathrm{CD}^{+} \mathrm{T}$ cells (125). Although there is no direct experimental evidence for this in humans, a better understanding of the immune responses to bacterial respiratory pathogens would open up new avenues for vaccine development.

An alternative approach to prevent pneumococcal pneumonia in young infants is to immunize the mother and increase the level of pneumococcal-specific antibody passed to the baby via either the placenta or breast milk. Researchers analyzed the outcomes of infants from Papua New Guinea whose mothers had enrolled into a trial of a 14-valent pneumococcal polysaccharide vaccine around the time of birth (124). In children who were aged 1-17 months when their mothers enrolled, and therefore were probably breastfeeding, the incidence of pneumonia was substantially lower among children of vaccinated mothers than among children of mothers in the control group (254 vs. 303 per 1,000 person-years of observation; ref. 126). Subsequently, a number of studies have confirmed the safety of pneumococcal polysaccharide vaccines during pregnancy and documented useful levels of antibody in infant serum and breast milk (127). This remains a strategy with considerable potential for preventing pneumococcal pneumonia in young infants and is under active investigation in northern Australia.

Vaccination against pneumonia and bronchiolitis caused by infection with $R S V$. RSV is the pneumonia-causing pathogen that poses the most complex challenges for vaccine development. It causes a broad spectrum of disease severity from coryza (runny nose) through bronchiolitis to alveolar pneumonia in infants, especially those aged under 3 months. In The Gambia, RSV is detectable in onefifth of children admitted to hospitals with lower respiratory tract infections (LRTIs) (128). The annual incidence of severe pneumonia caused by RSV is 5-10 per 1,000 children under 5 years of age in several developing countries (128-130), and an effective RSV vaccine could have substantial impact on childhood pneumonia.
Unfortunately, unexpected results from early studies have severely hampered the development of RSV vaccines $(131,132)$. A vaccine containing formalin-inactivated RSV administered to children in the 1960s not only failed to protect against infection, but enhanced the frequency and severity of pulmonary disease following natural infection (132). In one study, nearly $80 \%$ of vaccinees were hospitalized with a LRTI compared with $5 \%$ of control children (132). Furthermore, immunity following clearance of a RSV infection does not prevent reinfection, although it does lead to a reduction in the severity of illness and a switch from lower to upper respiratory tract disease $(133,134)$. Immunity is both humoral and cell mediated at different stages of infection; neutralizing antibodies specific for the F and $\mathrm{G}$ glycoproteins protect against infection, and RSV-specific CTLs terminate an infective episode. Natural exposure to RSV stimulates a balanced $\mathrm{CD}^{+}{ }^{+}$Th cell response with both proinflammatory Th1 cells (secreting IFN- $\gamma$ ) and antiinflammatory Th2 cells (secreting IL-10). However, one hypothesis explaining disease enhancement among the infants administered the vaccine containing formalin-inactivated RSV is that the vaccine biased the immune system to produce a predominantly Th2 response upon later exposure to virus (135). Ideally, vaccines against RSV disease should induce the same balanced Th1/Th2 response of natural immunity. They should also cover the antigenically distinct groups RSV A and RSV B.

Despite this immunological challenge, there are optimistic developments, particularly with subunit (purified F glycoprotein, $\mathrm{G}$ glycoprotein, and matrix proteins) and live-attenuated vaccines. A cold-passaged ( $c p$ ) derivative of RSV, which caused mild respiratory illness in young children, has been attenuated further by chemical mutagenesis to produce temperature sensitive (ts) strains of RSV (136). One of these, cpts248/404, which was highly attenuated in RSV-seronegative children, proved immunogenic when delivered intranasally to young infants (1-2 months old) but caused unacceptable nasal congestion (137). In an iterative testing process, recombinant technology is being used to manipulate this strain to develop a vaccine that is hoped will have optimal immunogenicity and acceptability (138).

\section{Management of pneumonia cases}

The effective management of cases of pneumonia relies on appropriate antibiotic therapy and supportive care, particularly with oxygen in more severe cases. The WHO has promoted case management at the level of community health worker and first-level health facility (139). Initial treatment is with either cotrimoxazole or amoxicillin. Those with lower chest wall indrawing or signs of severe disease, such as convulsions or an inability to drink, are referred to a hospital for inpatient care. A meta-analysis of field trials of the introduction of pneumonia treatment in the community, implemented by simply trained health workers using oral antibiotics, concluded that the intervention was associated with a surprising $20 \%$ impact on infant mortality of any cause and a greater impact on mortality in neonates and children $1-5$ years old (140). However, this impact was seen in settings where access to antibiotics was very limited prior to the intervention; such an impact cannot be generalized to settings where antibiotic therapy is already widely available.

Since 1992, the management of cases of childhood pneumonia has been incorporated into a broader strategy known as Integrated Management of Childhood Illness (IMCI), which was developed by the WHO and is now the standard of care for sick children in most 
developing countries. So far, IMCI has only been introduced into communities that already have access to health care, thus excluding children whose risk of mortality is highest (141). Children in the poorest communities are at the highest risk of developing conditions such as pneumonia, yet they are least likely to be able to access interventions, leading to inequity - unfair inequality - in child survival. By training community health workers to implement IMCI, the WHO aims to extend its reach beyond existing health services and to improve equity of access. Work in the field of equity has largely concentrated on analyses of access to interventions and on mortality risk by wealth quintiles (142), comparing the richest quintile of a population with the poorest. In many settings, this fails to identify the main determinants of risk, which might be geographic or ethnographic rather than financial. Further research is needed to define the parameters of equity and develop systems to monitor changes.

The impact of antimicrobial resistance on the management of childhood pneumonia is unclear. Rates of resistance to cotrimoxazole among strains of S. pneumoniae and $H$. influenzae are variable but generally very high, particularly in southern Africa, where many children and adults infected with HIV now receive longterm treatment with cotrimoxazole to prevent pneumonia caused by Pneumocystis jirovecii $(143,144)$. Although studies have repeatedly failed to establish a link between cotrimoxazole resistance and failure to respond to treatment for pneumonia $(145,146)$, amoxicillin is now preferred as a first-line treatment for pneumonia, based in part on the finding that more severe cases do better with amoxicillin (145).

Unfortunately, penicillin resistance, which implies amoxicillin resistance, is also becoming widespread among strains of S. pneumoniae. Some studies from Asia, Africa, and the Middle East have reported some degree of resistance in 50\% of strains (147), although others have reported a somewhat lower prevalence (143). The proportion of children with pneumonia caused by resistant strains might fall with the introduction PCVs, but the effect is likely to be temporary $(148,149)$. Evidence from studies of middle ear infections (150) shows that amoxicillin is effective in most cases caused by penicillin-resistant pneumococci, although the rate of bacteriological failure (persistent infection after a course of antibiotics) is higher than with penicillin-sensitive isolates. Amoxicillin therefore remains the antibiotic of choice for the treatment of pneumonia, although higher doses might be required in settings with high levels of penicillin insensitivity, stressing the importance of local and regional surveillance for antibiotic resistance.

Much of the mortality saving that can be achieved by case management of pneumonia through IMCI depends on the identification of severe cases for hospital referral. Unfortunately, the quality of inpatient care in developing countries is highly variable, leading to high rates of fatality from severe pneumonia in the poorest hospitals (151). This might be attributable to inadequate delivery of oxygen and antibiotics as well as to complications such as nosocomial infection or intravenous line problems. The recent finding that oral amoxicillin is equivalent to parenteral antibiotics for the management of severe pneumonia has prompted some agencies to recommend home treatment of severe pneumonia (152). However, this ignores the potential benefits of oxygen and other supportive therapy that are available only in hospitals and cannot yet be generally recommended. Meanwhile, efforts to improve the quality of inpatient pediatric care, currently being led by the WHO, are essential to reduce mortality in childhood pneumonia.

\section{Conclusions}

Pneumonia is a global disease that is typically curable in developed countries but often fatal in developing countries. The global perception of pneumonia as a public health problem is emasculated by its familiar and benign image in the industrialized world. Pneumonia has no UN agency to highlight its importance and no fund or series of global networks to advocate for drugs, vaccines, or care. The ambiguity of its various acronyms (e.g., ARI and LRTI) further undermines its perception as a single, tractable problem. The vigorous response to SARS and the considerable support for pandemic influenza preparedness ( $\$ 10$ billion) by the global public health community demonstrate what can be achieved when the world perceives a substantial health threat. Pneumonia, with a global burden of 5,000 childhood deaths every day, is a continuous, tangible threat that should trigger similar responses - action and research on pneumonia are urgently required.

Research on pneumonia is also very likely to be effective, especially if the problem is tackled comprehensively. A far-reaching approach should include (a) a better understanding of the etiology to help guide vaccine targets and antimicrobial therapy and to identify important atypical causes such as M. tuberculosis; (b) studies of pathogenesis and host response to improve case management and clinical outcome; (c) development of surveillance tools with new diagnostics to distinguish epidemics and identify novel pathogens; and (d) community-based studies to evaluate the impact of prevention and case management strategies, to describe the epidemiology of fatal pneumonia, and to investigate the role of equity in death from pneumonia in childhood.

\section{Acknowledgments}

J.A.G. Scott is funded by the Wellcome Trust of Great Britain through a senior fellowship in clinical science (no. 061089). J.S.M. Peiris is funded by Central Allocation Grant HKU1/05C from the Research Grants Council of Hong Kong. The authors are grateful to Angela Hwang of the Bill and Melinda Gates Foundation for help with the preparation of the manuscript.

Address correspondence to: J. Anthony G. Scott, Wellcome Trust/ KEMRI Collaborative Research Programme, PO Box 230, Kilifi 80108, Kenya. Phone: 254-41-522063; Fax: 254-41-522390; E-mail: ascott@ikilifi.net.

\footnotetext{
1. Williams, B.G., Gouws, E., Boschi-Pinto, C., Bryce, J., and Dye, C. 2002. Estimates of world-wide distribution of child deaths from acute respiratory infections. Lancet Infect. Dis. 2:25-32.

2. WHO Programme for Control of Acute Respiratory Infections. 1990. Acute respiratory infections in children: case management in small hospitals in developing countries. A manual for doctors and other senior health workers. WHO. Geneva, Swit-
}

zerland. $74 \mathrm{pp}$.

3. Scott, J.A., et al. 2000. Aetiology, outcome, and risk factors for mortality among adults with acute pneumonia in Kenya. Lancet. 355:1225-1230.

4. Metlay, J.P., Fishman, N.O., Joffe, M., and Edelstein, P.H. 2006. Impact of pediatric vaccination with pneumococcal conjugate vaccine on the risk of bacteremic pneumococcal pneumonia in adults. Vaccine. 24:468-475.
5. Preston, S.R., and Haines, M.R. 1991. Fatal years child mortality in late 19th century America. Princeton University Press. Princeton, New Jersey, USA. 4-5.

6. Grove, R.D., and Hetzel, A.M. 1968. Vital statistics rates in the United States, 1940-1960. US Department of Health, Education and Welfare, Public Health Service. Washington, DC, USA. 478-479.

7. Linder, F.E., and Grove, R.D. 1947. Vital statistics rates in the United States, 1900-1940. United States 
Government Printing Office. Washington, DC, USA. 254-255.

8. Mulholland, K. 2007. Perspectives on the burden of pneumonia in children. Vaccine. 25:2394-2397.

9. Rudan, I., Tomaskovic, L., Boschi-Pinto, C., and Campbell, H. 2004. Global estimate of the incidence of clinical pneumonia among children under five years of age. Bull. World Health Organ. 82:895-903.

10. UNICEF. 2007. State of the World's Children. New York: United Nations Children's Fund.

11. Cherian, T., et al. 2005. Standardized interpretation of paediatric chest radiographs for the diagnosis of pneumonia in epidemiological studies. Bull. World Health Organ. 83:353-359.

12. Cutts, F.T., et al. 2005. Efficacy of nine-valent pneumococcal conjugate vaccine against pneumonia and invasive pneumococcal disease in The Gambia: randomised, double-blind, placebo-controlled trial. Lancet. 365:1139-1146.

13. Scott, J.A., and Hall, A.J. 1999. The value and complications of percutaneous transthoracic lung aspiration for the etiologic diagnosis of community-acquired pneumonia. Chest. 116:1716-1732.

14. Shann, F., et al. 1984. Aetiology of pneumonia in children in Goroka Hospital, Papua New Guinea. Lancet. 2:537-541.

15. Adegbola, R.A., et al. 1994. The etiology of pneumonia in malnourished and well-nourished Gambian children. Pediatr. Infect. Dis. J. 13:975-982.

16. Lee, W.M., et al. 2007. High-throughput, sensitive, and accurate multiplex PCR-Microsphere flow cytometry system for large-scale comprehensive detection of respiratory viruses. J. Clin. Microbiol. 45:2626-2634.

17. Fan, J., Henrickson, K.J., and Savatski, L.L. 1998 Rapid simultaneous diagnosis of infections with respiratory syncytial viruses $A$ and $B$, influenza viruses $A$ and $B$, and human parainfluenza virus types 1,2 , and 3 by multiplex quantitative reverse transcription-polymerase chain reaction-enzyme hybridization assay (Hexaplex). Clin. Infect. Dis. 26:1397-1402.

18. Coiras, M.T., Aguilar, J.C., Garcia, M.L., Casas, I., and Perez-Brena, P. 2004. Simultaneous detection of fourteen respiratory viruses in clinical specimens by two multiplex reverse transcription nested-PCR assays. J. Med. Virol. 72:484-495.

19. Syrmis, M.W., et al. 2004. A sensitive, specific, and cost-effective multiplex reverse transcriptase-PCR assay for the detection of seven common respiratory viruses in respiratory samples. J. Mol. Diagn. 6:125-131.

20. Templeton, K.E., Scheltinga, S.A., Beersma, M.F.C., Kroes, A.C.M., and Claas, E.C.J. 2004. Rapid and sensitive method using multiplex real-time PCR for diagnosis of infections by influenza a and influenza $B$ viruses, respiratory syncytial virus, and parainfluenza viruses 1, 2, 3, and 4. J. Clin. Microbiol. 42:1564-1569.

21. Khanna, M., et al. 2005. The pneumoplex assays, a multiplex PCR-enzyme hybridization assay that allows simultaneous detection of five organisms, Mycoplasma pneumoniae, Chlamydia (Chlamydophila) pneumoniae, Legionella pneumophila, Legionella micdadei, and Bordetella pertussis, and its real-time counterpart. J. Clin. Microbiol. 43:565-571.

22. Mahony, J., et al. 2007. Development of a Respiratory Virus Panel (RVP) test for the detection of twenty human respiratory viruses using Multiplex PCR and a fluid microbead-based assay. J. Clin. Microbiol. 45:2965-2970.

23. Briese, T., et al. 2005. Diagnostic system for rapid and sensitive differential detection of pathogens. Emerg. Infect. Dis. 11:310-313.

24. Wang, D., et al. 2002. Microarray-based detection and genotyping of viral pathogens. Proc. Natl. Acad. Sci. U. S. A. 99:15687-15692.
25. Ecker, D.J., et al. 2005. Rapid identification and strain-typing of respiratory pathogens for epidemic surveillance. Proc. Natl. Acad. Sci. U. S. A. 102:8012-8017.

26. Chiu, C.Y., et al. 2007. Diagnosis of a critical respiratory illness caused by human metapneumovirus by use of a pan-virus microarray. J. Clin. Microbiol. 45:2340-2343

27. Palacios, G., et al. 2007. Panmicrobial oligonucleotide array for diagnosis of infectious diseases. Emerg. Infect. Dis. 13:73-81.

28. Murdoch, D.R., et al. 2001. Evaluation of a rapid immunochromatographic test for detection of Streptococcus pneumoniae antigen in urine samples from adults with community-acquired pneumonia. J. Clin. Microbiol. 39:3495-3498.

29. Leeming, J.P., Cartwright, K., Morris, R., Martin, S.A., and Smith, M.D. 2005. Diagnosis of invasive pneumococcal infection by serotype-specific urinary antigen detection. J. Clin. Microbiol. 43:4972-4976.

30. Dowell, S.F., Garman, R.L., Liu, G., Levine, O.S. and Yang, Y.H. 2001. Evaluation of Binax NOW, an assay for the detection of pneumococcal antigen in urine samples, performed among pediatric patients. Clin. Infect. Dis. 32:824-825.

31. Hill, P.C., et al. 2006. Nasopharyngeal carriage of Streptococcus pneumoniae in Gambian villagers. Clin. Infect. Dis. 43:673-679.

32. Korppi, M. 2004. Non-specific host response markers in the differentiation between pneumococcal and viral pneumonia: what is the most accurate combination? Pediatr. Int. 46:545-550.

33. Simon, L., Gauvin, F., Amre, D.K., Saint-Louis, P., and Lacroix, J. 2004. Serum procalcitonin and C-reactive protein levels as markers of bacterial infection: a systematic review and meta-analysis. Clin. Infect. Dis. 39:206-217.

34. van der Meer, V., Neven, A.K., van den Broek, P.J., and Assendelft, W.J. 2005. Diagnostic value of C reactive protein in infections of the lower respiratory tract: systematic review. BMJ. 331:26.

35. Madhi, S.A., Kohler, M., Kuwanda, L., Cutland, C., and Klugman, K.P. 2006. Usefulness of C-reactive protein to define pneumococcal conjugate vaccine efficacy in the prevention of pneumonia. Pediatr. Infect. Dis. J. 25:30-36.

36. Pauksen, K., Elfman, L., Ulfgren, A.K., and Venge, P. 1994. Serum levels of granulocyte-colony stimulating factor (G-CSF) in bacterial and viral infections, and in atypical pneumonia. Br. J. Haematol. 88:256-260.

37. Kragsbjerg, P., Jones, I., Vikerfors, T., and Holmberg, H. 1995. Diagnostic value of blood cytokine concentrations in acute pneumonia. Thorax. 50:1253-1257.

38. Ortqvist, A., Hedlund, J., Wretlind, B., Carlstrom, A., and Kalin, M. 1995. Diagnostic and prognostic value of interleukin- 6 and C-reactive protein in community-acquired pneumonia. Scand. J. Infect. Dis. 27:457-462.

39. Kennon, C., et al. 1996. Granulocyte colony-stimulating factor as a marker for bacterial infection in neonates. J. Pediatr. 128:765-769.

40. Fischer, J.E., Benn, A., Harbarth, S., Nadal, D., and Fanconi, S. 2002. Diagnostic accuracy of G-CSF, IL-8, and IL-1ra in critically ill children with suspected infection. Intensive Care Med. 28:1324-1331.

41. Gibot, S., et al. 2004. Soluble triggering receptor expressed on myeloid cells and the diagnosis of pneumonia. N. Engl. J. Med. 350:451-458.

42. Neuzil, K.M., Mellen, B.G., Wright, P.F., Mitchel, E.F., Jr., and Griffin, M.R. 2000. The effect of influenza on hospitalizations, outpatient visits, and courses of antibiotics in children. N. Engl. J. Med. 342:225-231.

43. Chiu, S.S., Lau, Y.L., Chan, K.H., Wong, W.H.S., and Peiris, J.S.M. 2002. Influenza-related hospitalizations among children in Hong Kong. N. Engl. J.
Med. 347:2097-2103.

44. Katz, M.A., et al. 2007. Who gets hospitalized for influenza pneumonia in Thailand? Implications for vaccine policy. Vaccine. 25:3827-3833.

45. Schwarzmann, S.W., Adler, J.L., Sullivan, R.J., Jr., and Marine, W.M. 1971. Bacterial pneumonia during the Hong Kong influenza epidemic of 1968-1969. Arch. Intern. Med. 127:1037-1041.

46. O’Brien, K.L., et al. 2000. Severe pneumococcal pneumonia in previously healthy children: the role of preceding influenza infection. Clin. Infect. Dis. 30:784-789.

47. McCullers, J.A. 2006. Insights into the interaction between influenza virus and pneumococcus. Clin. Microbiol. Rev. 19:571-582.

48. Madhi, S.A., and Klugman, K.P. 2004. A role for Streptococcus pneumoniae in virus-associated pneumonia. Nat. Med. 10:811-813.

49. Jennings, L.C., Anderson, T.P., Werno, A.M., Beynon, K.A., and Murdoch, D.R. 2004. Viral etiology of acute respiratory tract infections in children presenting to hospital: role of polymerase chain reaction and demonstration of multiple infections. Pediatr. Infect. Dis. J. 23:1003-1007.

50. Templeton, K.E., et al. 2005. Improved diagnosis of the etiology of community-acquired pneumonia with real-time polymerase chain reaction. Clin. Infect. Dis. 41:345-351.

51. van der Hoek, L., et al. 2004. Identification of a new human coronavirus. Nat. Med. 10:368-373.

52. Woo, P.C., et al. 2005. Characterization and complete genome sequence of a novel coronavirus, coronavirus HKU1, from patients with pneumonia. J. Virol. 79:884-895.

53. Lamson, D., et al. 2006. MassTag polymerasechain-reaction detection of respiratory pathogens, including a new rhinovirus genotype, that caused influenza-like illness in New York State during 2004-2005. J. Infect. Dis. 194:1398-1402.

54. Leung, C.-W., et al. 2004. Severe acute respiratory syndrome among children. Pediatrics. 113:e535-e543.

55. Wong, G.W.K., Li, A.M., Ng, P.C., and Fok, T.F. 2003. Severe acute respiratory syndrome in children. Pediatr. Pulmonol. 36:261-266.

56. Chen, J.-M., Chen, J.-W., Dai, J.-J., and Sun, Y.-X. 2007. A survey of human cases of H5N1 avian influenza reported by the WHO before June 2006 for infection control. Am. J. Infect. Control. 35:351-353.

57. Lipsitch, M., et al. 2003. Transmission dynamics and control of severe acute respiratory syndrome. Science. 300:1966-1970.

58. Gillim-Ross, L., and Subbarao, K. 2006. Emerging respiratory viruses: challenges and vaccine strategies. Clin. Microbiol. Rev. 19:614-636.

59. Yang, Z.-Y., et al. 2005. Evasion of antibody neutralization in emerging severe acute respiratory syndrome coronaviruses. Proc. Natl. Acad. Sci. U. S. A. 102:797-801.

60. Bell, D.M. 2006. Non-pharmaceutical interventions for pandemic influenza, international measures. Emerg. Infect. Dis. 12:81-87.

61. Fox, J.P., and Hall, C.E. 1980. Viruses in families: surveillance of families as a key to epidemiology of virus infection. PSG Publishing Company. Littleton, Massachusetts, USA. 441 pp.

62. Lloyd-Smith, J.O., Schreiber, S.J., Kopp, P.E., and Getz, W.M. 2005. Superspreading and the effect of individual variation on disease emergence. Nature. 438:355-359.

63. Weber, M.W., Palmer, A., Oparaugo, A., and Mulholland, E.K. 1995. Comparison of nasal prongs and nasopharyngeal catheter for the delivery of oxygen in children with hypoxemia because of a lower respiratory tract infection. J. Pediatr. 127:378-383

64. Onyango, F.E., et al. 1993. Hypoxaemia in young Kenyan children with acute lower respiratory infection. BMJ. 306:612-615. 
65. Smyth, A., Carty, H., and Hart, C.A. 1998. Clinical predictors of hypoxaemia in children with pneumonia. Ann. Trop. Paediatr. 18:31-40.

66. Duke, T., Mgone, J., and Frank, D. 2001. Hypoxaemia in children with severe pneumonia in Papua New Guinea. Int. J. Tuberc. Lung Dis. 5:511-519.

67. [Anonymous]. 2004. Informal consultation on clinical use of oxygen. Meeting report 2-3 October 2003. Child and Adolescent Health and Development. WHO. Geneva, Switzerland. 18 pp.

68. Wandi, F., Peel, D., and Duke, T. 2006. Hypoxaemia among children in rural hospitals in Papua New Guinea: epidemiology and resource availability - a study to support a national oxygen programme. Ann. Trop. Paediatr. 26:277-284.

69. L'Her, P., et al. 2006. The problem of oxygen in developing countries [In French]. Med. Trop. (Mars). 66:631-638.

70. Sommer, A., Katz, J., and Tarwotjo, I. 1984. Increased risk of respiratory disease and diarrhea in children with preexisting mild vitamin A deficiency. Am. J. Clin. Nutr. 40:1090-1095.

71. Bloem, M.W., et al. 1990. Mild vitamin A deficiency and risk of respiratory tract diseases and diarrhea in preschool and school children in northeastern Thailand. Am. J. Epidemiol. 131:332-339.

72. Huiming, Y., Chaomin, W., and Meng, M. 2005. Vitamin A for treating measles in children. Cochrane Database Syst. Rev. CD001479.

73. Brown, N., and Roberts, C. 2004. Vitamin A for acute respiratory infection in developing countries: a meta-analysis. Acta Paediatr. 93:1437-1442.

74. The Vitamin A and Pneumonia Working Group. 1995. Potential interventions for the prevention of childhood pneumonia in developing countries: a meta-analysis of data from field trials to assess the impact of vitamin A supplementation on pneumonia morbidity and mortality. Bull. World Health Organ. 73:609-619.

75. Grotto, I., Mimouni, M., Gdalevich, M., and Mimouni, D. 2003. Vitamin A supplementation and childhood morbidity from diarrhea and respiratory infections: a meta-analysis. J. Pediatr. 142:297-304

76. Black, R.E. 2003. Zinc deficiency, infectious disease and mortality in the developing world. J. Nutr. 133:1485S-1489S.

77. Bhutta, Z.A., et al. 1999. Prevention of diarrhea and pneumonia by zinc supplementation in children in developing countries: pooled analysis of randomized controlled trials. Zinc Investigators' Collaborative Group. J. Pediatr. 135:689-697.

78. Brooks, W.A., et al. 2004. Zinc for severe pneumonia in very young children: double-blind placebocontrolled trial. Lancet. 363:1683-1688.

79. Brooks, W.A., et al. 2005. Effect of weekly zinc supplements on incidence of pneumonia and diarrhoea in children younger than 2 years in an urban, low-income population in Bangladesh: randomised controlled trial. Lancet. 366:999-1004.

80. Baqui, A.H., et al. 2002. Effect of zinc supplementation started during diarrhoea on morbidity and mortality in Bangladeshi children: community randomised trial. BMJ. 325:1059.

81. Gaetke, L.M., McClain, C.J., Talwalkar, R.T., and Shedlofsky, S.I. 1997. Effects of endotoxin on zinc metabolism in human volunteers. Am. J. Physiol. 272:E952-E956.

82. Cousins, R.J. 1985. Absorption, transport, and hepatic metabolism of copper and zinc: special reference to metallothionein and ceruloplasmin. Physiol. Rev. 65:238-309.

83. Truong-Tran, A.Q., et al. 2002. Altered zinc homeostasis and caspase- 3 activity in murine allergic airway inflammation. Am. J. Respir. Cell Mol. Biol. 27:286-296.

84. Cho, Y.H., et al. 2002. Antibacterial effect of intraprostatic zinc injection in a rat model of chronic bacterial prostatitis. Int. J. Antimicrob Agents. 19:576-582.

85. Makonnen, B., Venter, A., and Joubert, G. 2003. A randomized controlled study of the impact of dietary zinc supplementation in the management of children with protein-energy malnutrition in Lesotho. I: Mortality and morbidity. J. Trop. Pediatr. 49:340-352.

86. Sazawal, S., et al. 2007. Effect of zinc supplementation on mortality in children aged 1-48 months: a community-based randomised placebo-controlled trial. Lancet. 369:927-934.

87. Bobat, R., et al. 2005. Safety and efficacy of zinc supplementation for children with HIV-1 infection in South Africa: a randomised double-blind placebo-controlled trial. Lancet. 366:1862-1867.

88. Bose, A., et al. 2006. Efficacy of zinc in the treatment of severe pneumonia in hospitalized children $<2$ y old. Am. J. Clin. Nutr. 83:1089-1096.

89. Howie, S., Zaman, S.M.A., Omoruyi, O., Adegbola, R., and Prentice, A. 2007. Severe pneumonia research and the problem of case definition: the example of zinc trials. Am. J. Clin. Nutr. 85:242-243.

90. Mahalanabis, D., et al. 2002. Zinc supplementation as adjunct therapy in children with measles accompanied by pneumonia: a double-blind, randomized controlled trial. Am. J. Clin. Nutr. 76:604-607.

91. Mahalanabis, D., et al. 2004. Randomized, doubleblind, placebo-controlled clinical trial of the efficacy of treatment with zinc or vitamin A in infants and young children with severe acute lower respiratory infection. Am. J. Clin. Nutr. 79:430-436.

92. Knapp, S., et al. 2004. Toll-like receptor 2 plays a role in the early inflammatory response to murine pneumococcal pneumonia but does not contribute to antibacterial defense. J. Immunol. 172:3132-3138.

93. Lee, J.S., et al. 2005. TLR-4 pathway mediates the inflammatory response but not bacterial elimination in E. coli pneumonia. Am. J. Physiol. Lung Cell Mol. Physiol. 289:L731-L738.

94. Alexopoulou, L., Holt, A.C., Medzhitov, R., and Flavell, R.A. 2001. Recognition of double-stranded RNA and activation of NF-kappaB by Toll-like receptor 3. Nature. 413:732-738.

95. Miettinen, M., Sareneva, T., Julkunen, I., and Matikainen, S. 2001. IFNs activate toll-like receptor gene expression in viral infections. Genes Immun. 2:349-355.

96. Monton, C., and Torres, A. 1998. Lung inflammatory response in pneumonia. Monaldi Arch. Chest Dis. 53:56-63.

97. Fernandez-Serrano, S., et al. 2003. Molecular inflammatory responses measured in blood of patients with severe community-acquired pneumonia. Clin. Diagn. Lab. Immunol. 10:813-820.

98. Renckens, R., et al. 2006. The acute-phase response and serum amyloid $\mathrm{A}$ inhibit the inflammatory response to Acinetobacter baumannii Pneumonia. J. Infect. Dis. 193:187-195.

99. Cheung, C.Y., et al. 2002. Induction of proinflammatory cytokines in human macrophages by influenza A (H5N1) viruses: a mechanism for the unusual severity of human disease? Lancet. 360:1831-1837.

100.Kobasa, D., et al. 2007. Aberrant innate immune response in lethal infection of macaques with the 1918 influenza virus. Nature. 445:319-323.

101.Belshe, R.B., et al. 2004. Evaluation of combined live, attenuated respiratory syncytial virus and parainfluenza 3 virus vaccines in infants and young children. J. Infect. Dis. 190:2096-2103.

102.Kuklin, N.A., et al. 2006. A novel Staphylococcus aureus vaccine: iron surface determinant $B$ induces rapid antibody responses in rhesus macaques and specific increased survival in a murine $S$. aureus sepsis model. Infect. Immun. 74:2215-2223.

103.Andersen, P. 2007. Tuberculosis vaccines - an update. Nat. Rev. Microbiol. 5:484-487.
104.Barrett, D.J. 1985. Human immune responses to polysaccharide antigens: an analysis of bacterial polysaccharide vaccines in infants. Adv. Pediatr. 32:139-158.

105.Rijkers, G.T., Sanders, E.A., Breukels, M.A., and Zegers, B.J. 1998. Infant B cell responses to polysaccharide determinants. Vaccine. 16:1396-1400.

106.Douglas, R.M., Paton, J.C., Duncan, S.J., and Hansman, D.J. 1983. Antibody response to pneumococcal vaccination in children younger than five years of age. J. Infect. Dis. 148:131-137.

107. Avery, O.T., and Goebel, W.F. 1929. Chemo-immunological studies on conjugated carbohydrate-proteins: II. Immunological specificity of synthetic sugar-protein antigens. J. Exp. Med. 50:533-550.

108. Bisgard, K.M., et al. 1998. Haemophilus influenzae invasive disease in the United States, 1994-1995: near disappearance of a vaccine-preventable childhood disease. Emerg. Infect. Dis. 4:229-237.

109.[Anonymous]. 2005. Direct and indirect effects of routine vaccination of children with 7 -valent pneumococcal conjugate vaccine on incidence of invasive pneumococcal disease - United States, 1998-2003. MMWR Morb. Mortal Wkly. Rep. 54:893-897.

110.Adegbola, R.A., et al. 2005. Elimination of Haemophilus influenzae type $\mathrm{b}(\mathrm{Hib})$ disease from The Gambia after the introduction of routine immunisation with a Hib conjugate vaccine: a prospective study. Lancet. 366:144-150.

111.Wall, R.A., Corrah, P.T., Mabey, D.C., and Greenwood, B.M. 1986. The etiology of lobar pneumonia in the Gambia. Bull. World Health Organ. 64:553-558.

112.Forgie, I.M., et al. 1991. Etiology of acute lower respiratory tract infections in Gambian children: I. Acute lower respiratory tract infections in infants presenting at the hospital. Pediatr. Infect. Dis. J. 10:33-41.

113. Mulholland, K., et al. 1997. Randomised trial of Haemophilus influenzae type-b tetanus protein conjugate vaccine for prevention of pneumonia and meningitis in Gambian infants. Lancet. 349:1191-1197.

114.Jodar, L., et al. 2003. Serological criteria for evaluation and licensure of new pneumococcal conjugate vaccine formulations for use in infants. Vaccine. 21:3265-3272.

115.Mbelle, N., et al. 1999. Immunogenicity and impact on nasopharyngeal carriage of a nonavalent pneumococcal conjugate vaccine. J. Infect. Dis. 180:1171-1176.

116.Goldblatt, D., et al. 2005. Antibody responses to nasopharyngeal carriage of Streptococcus pneumoniae in adults: A longitudinal household study. J. Infect. Dis. 192:387-393.

117. Dagan, R., et al. 2002. Reduction of nasopharyngeal carriage of Streptococcus pneumoniae after administration of a 9-valent pneumococcal conjugate vaccine to toddlers attending day care centers. J. Infect. Dis. 185:927-936.

118.Singleton, R.J., et al. 2007. Invasive pneumococcal disease caused by nonvaccine serotypes among alaska native children with high levels of 7 -valent pneumococcal conjugate vaccine coverage. JAMA. 297:1784-1792.

119.Laine, C., et al. 2004. Age-specific immunoglobulin $\mathrm{g}(\mathrm{IgG})$ and $\operatorname{IgA}$ to pneumococcal protein antigens in a population in coastal Kenya. Infect. Immun. 72:3331-3335.

120. Hamel, J., et al. 2004. Prevention of pneumococcal disease in mice immunized with conserved surfaceaccessible proteins. Infect. Immun. 72:2659-2670.

121. Ogunniyi, A.D., Grabowicz, M., Briles, D.E., Cook, J., and Paton, J.C. 2007. Development of a vaccine against invasive pneumococcal disease based on combinations of virulence proteins of Streptococcus pneumoniae. Infect. Immun. 75:350-357.

122. Barocchi, M.A., et al. 2006. A pneumococcal pilus influences virulence and host inflammatory responses. Proc. Natl. Acad. Sci. U. S. A. 103:2857-2862. 
123. Briles, D.E., et al. 2000. Immunization of humans with recombinant pneumococcal surface protein A (rPspA) elicits antibodies that passively protect mice from fatal infection with Streptococcus pneumoniae bearing heterologous PspA. J. Infect. Dis. 182:1694-1701.

124.Malley, R., et al. 2001. Intranasal immunization with killed unencapsulated whole cells prevents colonization and invasive disease by capsulated pneumococci. Infect. Immun. 69:4870-4873.

125. Malley, R., et al. 2005. CD4+ T cells mediate antibody-independent acquired immunity to pneumococcal colonization. Proc. Natl. Acad. Sci. U. S. A. 102:4848-4853.

126.Lehmann, D., Pomat, W.S., Riley, I.D., and Alpers, M.P. 2003. Studies of maternal immunisation with pneumococcal polysaccharide vaccine in Papua New Guinea. Vaccine. 21:3446-3450.

127. [Anonymous]. 1998. Report of a meeting on maternal and neonatal pneumococcal immunization. January 26-27. WHO. Geneva, Switzerland. 61 pp.

128. Weber, M.W., et al. 2002. An epidemiological study of RSV infection in the Gambia. Bull. World Health Organ. 80:562-568.

129. Robertson, S.E., et al. 2004. Respiratory syncytial virus infection: denominator-based studies in Indonesia, Mozambique, Nigeria and South Africa. Bull. World Health Organ. 82:914-922.

130.Nokes, D.J., et al. 2007. Respiratory Syncytial Virus infection and disease in infants and young children studied from birth in Kilifi District, Kenya. Clin. Infect. Dis. 46:50-57.

131.Chin, J., Magoffin, R.L., Shearer, L.A., Schieble, J.H., and Lennette, E.H. 1969. Field evaluation of a respiratory syncytial virus vaccine and a trivalent parainfluenza virus vaccine in a pediatric population. Am. J. Epidemiol. 89:449-463.

132. Kim, H.W., et al. 1969. Respiratory syncytial virus disease in infants despite prior administration of antigenic inactivated vaccine. Am. J. Epidemiol. 89:422-434.

133.Hall, C.B., Walsh, E.E., Long, C.E., and Schnabel, K.C. 1991. Immunity to and frequency of reinfection with respiratory syncytial virus. J. Infect. Dis. 163:693-698.

134.Henderson, F.W., Collier, A.M., Clyde, W.A., Jr., and Denny, F.W. 1979. Respiratory-syncytial-virus infections, reinfections and immunity. A prospective, longitudinal study in young children. $N$. Engl. J. Med. 300:530-534.

135.van Drunen Littel-van den Hurk, S., Mapletoft,
J.W., Arsic, N., and Kovacs-Nolan, J. 2007. Immunopathology of RSV infection: prospects for developing vaccines without this complication. Rev. Med. Virol. 17:5-34.

136.Crowe, J.E., Jr., Bui, P.T., Davis, A.R., Chanock, R.M., and Murphy, B.R. 1994. A further attenuated derivative of a cold-passaged temperature-sensitive mutant of human respiratory syncytial virus retains immunogenicity and protective efficacy against wild-type challenge in seronegative chimpanzees. Vaccine. 12:783-790.

137. Wright, P.F., et al. 2000. Evaluation of a live, coldpassaged, temperature-sensitive, respiratory syncytial virus vaccine candidate in infancy. J. Infect. Dis. 182:1331-1342.

138. Karron, R.A., et al. 2005. Identification of a recombinant live attenuated respiratory syncytial virus vaccine candidate that is highly attenuated in infants. J. Infect. Dis. 191:1093-1104.

139.[Anonymous]. 2000. Handbook IMCI integrated management of childhood illness. Department of Child and Adolescent Health and Development. WHO. Geneva, Switzerland. 173 pp.

140. Sazawal, S., and Black, R.E. 2003. Effect of pneumonia case management on mortality in neonates, infants, and preschool children: a meta-analysis of community-based trials. Lancet Infect. Dis. 3:547-556.

141. Gwatkin, D.R. 2006. IMCI: what can we learn from an innovation that didn't reach the poor? Bull. World Health Organ. 84:768.

142.Victora, C.G., et al. 2003. Applying an equity lens to child health and mortality: more of the same is not enough. Lancet. 362:233-241.

143. Benbachir, M., et al. 2001. Two-year surveillance of antibiotic resistance in Streptococcus pneumoniae in four African cities. Antimicrob. Agents Chemother. 45:627-629.

144.Buie, K.A., et al. 2004. Gender as a risk factor for both antibiotic resistance and infection with pediatric serogroups/serotypes, in HIV-infected and -uninfected adults with pneumococcal bacteremia. J. Infect. Dis. 189:1996-2000.

145.Straus, W.L., et al. 1998. Antimicrobial resistance and clinical effectiveness of co-trimoxazole versus amoxycillin for pneumonia among children in Pakistan: randomised controlled trial. Lancet 352:270-274.

146. Catchup Study Group. 2002. Clinical efficacy of cotrimoxazole versus amoxicillin twice daily for treatment of pneumonia: a randomised controlled clinical trial in Pakistan. Arch. Dis. Child. 86:113-118.
147. Dobay, O., Rozgonyi, F., and Amyes, S. 2004. Virulence factors, antibiotic resistance mechanisms and the prevalence of resistance worldwide in Streptococcus pneumoniae. Rev. Med. Microbiol. 15:27-39.

148.Cohen, R., et al. 2006. Impact of Pneumococcal Conjugate Vaccine and of reduction of antibiotic use on nasopharyngeal carriage of nonsusceptible pneumococci in children with acute otitis media. Pediatr. Infect Dis. J. 25:1001-1007.

149. Messina, A.F., et al. 2007. Impact of the pneumococcal conjugate vaccine on serotype distribution and antimicrobial resistance of invasive Streptococcus pneumoniae isolates in Dallas, TX, children from 1999 through 2005. Pediatr. Infect. Dis. J. 26:461-467.

150.Dagan, R. 2000. Treatment of acute otitis media challenges in the era of antibiotic resistance. Vaccine. 19(Suppl. 1):S9-S16.

151.Nolan, T., et al. 2001. Quality of hospital care for seriously ill children in less-developed countries. Lancet. 357:106-110.

152.Addo-Yobo, E., et al. 2004. Oral amoxicillin versus injectable penicillin for severe pneumonia in children aged 3 to 59 months: a randomised multicentre equivalency study. Lancet. 364:1141-1148.

153.Falade, A.G., Mulholland, E.K., Adegbola, R.A., and Greenwood, B.M. 1997. Bacterial isolates from blood and lung aspirate cultures in Gambian children with lobar pneumonia. Ann. Trop. Paediatr. 17:315-319.

154.Forgie, I.M., et al. 1991. Etiology of acute lower respiratory tract infections in Gambian children: II. Acute lower respiratory tract infection in children ages one to nine years presenting at the hospital. Pediatr. Infect. Dis. J. 10:42-47.

155.Tran, T.T., et al. 1998. The etiology of bacterial pneumonia and meningitis in Vietnam. Pediatr. Infect. Dis. J. 17:S192-S194.

156.Selwyn, B.J. 1990. The epidemiology of acute respiratory tract infection in young children: comparison of findings from several developing countries. Coordinated Data Group of BOSTID Researchers. Rev. Infect. Dis. 12(Suppl. 8):S870-S888.

157. McNally, L.M., et al. 2007. Effect of age, polymicrobial disease, and maternal HIV status on treatment response and cause of severe pneumonia in South African children: a prospective descriptive study. Lancet. 369:1440-1451.

158.Mimica, I., Donoso, E., Howard, J.E., and Ledermann, G.W. 1971. Lung puncture in the etiological diagnosis of pneumonia. A study of 543 infants and children. Am. J. Dis. Child. 122:278-282. 